\title{
Study of Word Prediction for Utterance Support System
}

\author{
Yuya Hamai $^{\text {a, }}$, Mitsunori Mizumachi ${ }^{\mathrm{a}}$, Yoshihisa Nakatoh ${ }^{\mathrm{a}}$, Kenji Matsui $^{\mathrm{b}}$ \\ ${ }^{a}$ Kyushu Institute of Technology, 1-1 Sensui-cho, Tobata-ku, Kitakyushu 804-8550, Japan \\ ${ }^{\mathrm{b}}$ Osaka Institute of Technology, 5-16-1 Oomiya, Asahi-ku, Oosaka 535-0002, Japan \\ *Corresponding Author: n349427y@ tobata.isc.kyutech.ac.jp
}

\begin{abstract}
We studied new utterance support system working on an information terminal for people who have speech handicaps. We made word prediction and added fixed phrases from the dictionary including phrases often used in daily life conversations and class 2-gram using co-occurrence frequencies of parts of speech as additional functions to make it more effective and faster. And we did evaluation experiment with other methods.
\end{abstract}

Keywords: utterance support, word prediction, class 2-gram.

\section{Introduction}

Speech is very important for us to communicate with others in our daily lives. However, because of speech handicaps, some people find it difficult to do that. The main approaches for the people to communicate with others are writing and sign language. But they are difficult to master and writing needs to carry pens and papers at all times ${ }^{(1)}$. Nowadays, many new utterance supports method are studied. For example, "GlovalVoice voice support" is a communication tool works on $\mathrm{pad}^{(2)}$. The user inputs desired words and then pad outputs it as a voice by using voice synthesis.

Our main purpose is to study a new utterance support system using an information terminal (ex. Smartphone, pad). And to make this system faster and more effective, we studied a word input method and voice synthesis. We studied a word prediction, especially. It is one of word converting methods and it predicts user's desired words by using the small part of the word entered by the user ${ }^{(3)(4)}$. To make a word prediction faster and more effective, we used 1) the fixed phrases dictionary and 2) the class 2 -gram as additional functions. The fixed phrases dictionary includes phrases often used in daily life conversations and class 2-gram is 2-gram made from co-occurrence frequency of parts of speech (POS) in Japanese. And we did evaluation experiment to find the efficient of both fixed phrases dictionary and class 2-gram.

\section{Whole block diagram of the proposed utterance system}

This system consists of some parts in the whole block diagram (Figure 1).

The input part processes the Roman character codes generated by user's keyboard inputs, and then outputs them to word prediction part. Information terminals like smartphones have their own GUI and users generally use it to input words or phrases, but we used line Linux's input method and this might be more difficult than other input methods.

The word prediction part predicts words or phrases that user desired by using Roman character inputs processed in input part. The details of this part are shown in chapter 3.The voice synthesis part processes the words or phrases selected by user in the word prediction part and outputs them as voices. Some kinds of voices will be available and user can choose one that he likes. However, we didn't evaluate this part in this paper.

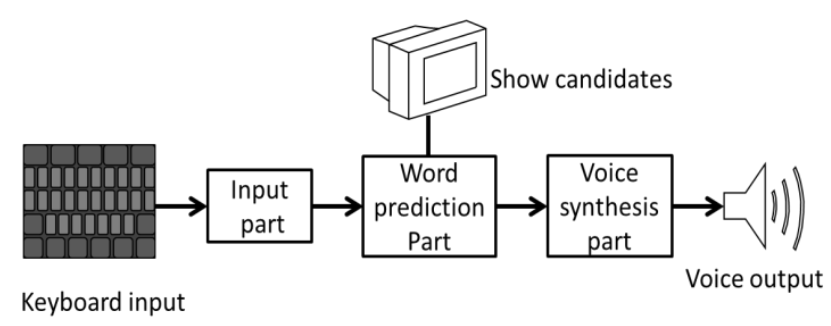

Figure 1. Whole block diagram 


\section{Detail of the word prediction part}

In this chapter, we show the details of the word prediction part. A word prediction is one of word converting methods and it predicts the desired words or phrases by small part of words entered by user. Nowadays this method is used in many information terminals having smaller number of keys. Figure 2 shows the detail of this part.

User's character inputs are used to search words or phrases in the dictionaries. The dictionaries have not only words or phrases but also POS and word frequencies with each of them and they are used to sort and predict candidate words or phrases. The sorting part sorts the candidates according to word and phrase frequencies and shows them to user for selection. The class 2-gram is used to change frequency and generate next candidates. The selector part shows the sorted candidates and the generated next candidates for user and the user choice his desired word from them. We show the details of 1) the dictionaries, 2) the word and phrase frequency and 3) the class 2-gram in following sections.

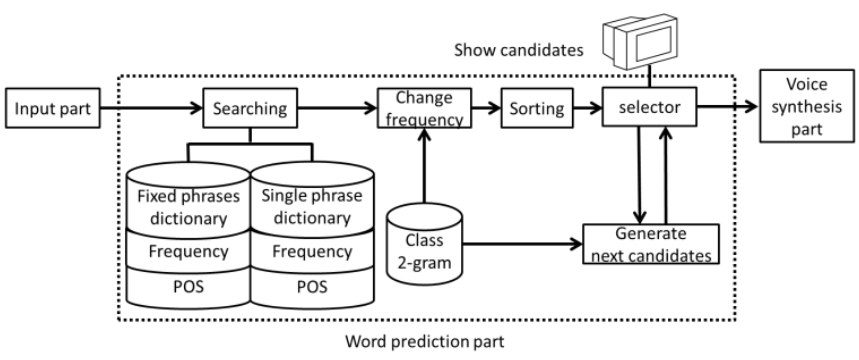

Figure 2. Word prediction part

\subsection{Dictionaries}

There are two dictionaries in this word prediction single phrase and fixed phrases - and user's input directly searches to both of them to show candidate words or phrases. We show the details of both dictionaries in following.

(a) Single phrase dictionary

We mainly used SKK dictionary (M size) ${ }^{(5)}$ to make this dictionary. The SKK dictionary has many nouns but other POS are not so much, thus we use a morphological analysis to Japanese example sentences in English conversation learning site by using Chasen ${ }^{(6)}$. Morphological analysis parses sentences into words and analyzes it in each POS. We added the words and POS in this dictionary and the total number of words in this dictionary became 13,798 .

The single phrase dictionary consists of 1) number, 2) pronunciation, 3 ) result word and 4) POS. Table 1 shows a concrete example of this dictionary. Pronunciation is used to search for words and all words are written in Roman characters. Roman character input can show candidates in smaller inputs and correct wrong spelling easier than Japanese. We pronounced the words using kakasi ${ }^{(7)}$ and was written in Kunrei-shiki. Result words were shown to user as search result. POS is used to change word frequency in dictionary and show next candidate words.

Table 1. Single phrase dictionary

\begin{tabular}{|c|c|c|c|}
\hline Number & Pronunciations & Result word & POS \\
\hline 5992 & katamuki & 傾き & Noun \\
\hline 5993 & katamusubi & 片結び & Noun \\
\hline 5994 & katame & 片目 & Noun \\
\hline$\ldots$ & $\ldots$ & $\ldots$ & $\cdots$ \\
\hline
\end{tabular}

(b) Fixed phrases dictionary

Fixed phrases dictionary consist of daily life conversation sentences and Table 2 shows that example. We made this dictionary to decrease user's inputs.

There are 550 phrases in this dictionary and they were from Japanese example sentences in Japanese learning sites for non-Japanese speakers. The structure of this dictionary is same as single phrase dictionary

Table 2. Fixed phrase dictionary

\begin{tabular}{|c|c|c|c|}
\hline Number & Pronunciation & Result word & POS \\
\hline 12 & gomennasai & ごめんなさい & Interjection \\
\hline 13 & suimasen & すいません & interjection \\
\hline 14 & mousiwakenai & 申し訳ない & Noun \\
\hline$\ldots$ & $\ldots$ & $\ldots$ & $\ldots$ \\
\hline
\end{tabular}

\subsection{Word and phrase frequency}

Word and phrase frequency changes its own values to learn user's habit. Table 3 shows an example. All of words and phrases in dictionaries have their own frequency. When a word or phrase was selected by user, the frequency which has same number increases one. If a desired word was not in sorted candidates, the user chose nothing from it and all of frequencies of words and phrases in candidates reduce their own frequency by half. After this operation, even user enters same character as latest entry, the candidates are refreshed and different words and phrases are shown up. 
Thus the probability of user's desired word or phrase coming up is increased. The frequency is also changed by an additional function of class 2-gram.

Table 3. Word and phrase frequencies

\begin{tabular}{|c|c|}
\hline Number & Frequency \\
\hline 0 & 1 \\
\hline 1 & 5 \\
\hline 2 & 8 \\
\hline$\cdots$ & $\cdots$ \\
\hline
\end{tabular}

\subsection{Class 2-gram}

(a) Class 2-gram

Class 2-gram is one kinds of 2-gram and is made from POS co-occurrence frequency ${ }^{(8)}$. We decided to use the class 2-gram in this word prediction because it has less data than 2-gram. Using 2-gram needs strong machine power because it has a lot of data. If there are 100 words in dictionary, it has $100 * 100$ data. However class 2-gram has only $9 * 9$ data of the number of words in its dictionary.

There are ten POS in Japanese and table 4 shows them. Although some people didn't recognize na-adjective as a POS, thus we use nine POS to make class 2-gram.

Table 4. Japanese POS

\begin{tabular}{|c|l|c|}
\hline \multirow{4}{*}{ Sort } & Independent words & Ancillary words \\
\hline \multirow{4}{*}{ term } & Verb & \multirow{2}{*}{ Modal verbs } \\
\cline { 2 - 2 } & Adjective & \\
\cline { 2 - 2 } & Na-adjective & \multirow{2}{*}{ Postpositional } \\
\hline \multirow{4}{*}{ predicate } & Noun & particle \\
\cline { 2 - 2 } & Adnominal adjective & \\
\cline { 2 - 2 } & Adverb & \\
\cline { 2 - 2 } & Conjunction & \\
\cline { 2 - 2 } & Interjection & \\
\hline
\end{tabular}

Figure 3 shows the way to make the class 2-gram. We made it by using 4000 Japanese example conversation texts and pick up POS and analysis their co-occurrence. Table 5 is an example of the class 2-gram. By using the table, two functions are addicted and they help word prediction to predict words and phrases more effectively.

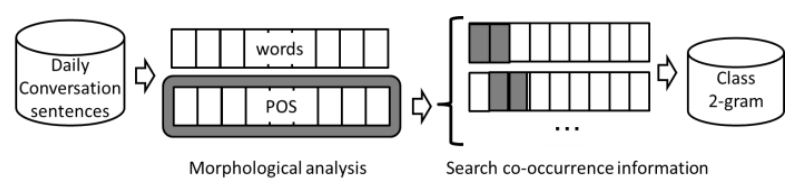

Figure 3. Way of making class 2-gram
Table 5. Examples of class 2-gram

\begin{tabular}{|c|c|c|c|}
\cline { 2 - 4 } \multicolumn{1}{c|}{} & Noun & Verb & Adjective \\
\hline Following POS & \multicolumn{3}{|c|}{ Probability[\%] } \\
\hline Conjunction & 0.061318 & 0.117256 & 0 \\
\hline Noun & 19.56055 & 13.30858 & 32.83396 \\
\hline Verb & 7.235565 & 7.777995 & 16.22971 \\
\hline Modal verbs & 11.17016 & 46.82431 & 27.8402 \\
\hline Interjection & 0.132856 & 0.175884 & 0 \\
\hline Postpositional particle & 60.98109 & 30.03713 & 20.09987 \\
\hline Adverb & 0.224834 & 0.390854 & 0.249688 \\
\hline Prefix & 0.194175 & 0.234512 & 0.249688 \\
\hline Adjective & 0.255493 & 0.605824 & 1.498127 \\
\hline Adnominal adjective & 0.183955 & 0.527653 & 0.998752 \\
\hline
\end{tabular}

(b) Next candidates

The next candidates are automatically predicted by using class 2-gram. The words or phrases have POS which are highest two of the class 2-gram and are automatically selected from dictionaries, sorted according to their frequencies and shown to users as next candidates. For example, if the user chooses a noun, next candidates are particle or noun.

(c) Frequency change

By using the latest result of the word prediction, word and phrase frequencies are changed by multiplying probabilities of the class 2-gram. For example, if the latest result was noun, then word and phrase frequencies of particle are multiplied by " 0.6 " and so are others. After this operation, all of POS except particle and noun become difficult to show up.

\section{Experiments}

We did experiments to find out our word prediction's performance by adding additional functions.

\subsection{Methods}

In this paper, we did two experiments - necessary numbers of input (/100 characters) and speed of input (/1 min) - and each result is shown in following sections. We especially paid attention to the efficiency of class 2 -gram. Sentence used in these experiences is from $\left\lceil 86^{\text {th }}\right.$ Examination in Japanese Word Processing $\rfloor$ and we compare our word prediction with the Microsoft Office IME 2010 (MS IME) and the iPhone. We use Wired Keyboard 600 to input character to the word prediction and MSIME. Table 6 shows the number of subjects of each experiment. 
Table 6. Number of subjects

\begin{tabular}{|l|l|}
\hline \multicolumn{1}{|c|}{ Evaluation } & Number of subjects \\
\hline Necessary numbers of input & 1 (author) \\
\hline Speed of input & 5 (including author) \\
\hline
\end{tabular}

\subsection{Results}

(a) Necessary numbers of input

Table 6 shows the experimental result of necessary numbers of input. There are two results of our system one is itself and other is with class 2-gram. According to Table 7, our word prediction needed less numbers or input than other methods. And adding class 2-gram makes it more effective and it has $40 \%$ lead over the iPhone.

Table 7. Result of necessary number of input

\begin{tabular}{|l|r|}
\hline \multicolumn{1}{|c|}{ Methods } & Numbers of input(/100 characters $)$ \\
\hline Our word prediction & 119 \\
\hline Ours + class 2-gram & 102 \\
\hline IPhone & 171 \\
\hline Microsoft Office IME & 221 \\
\hline
\end{tabular}

\section{(b) Speed of input}

Table 8 shows the result of speed of input. The values indicate how many characters subjects could enter and faster methods have bigger number. The results from the subjects show that using our word prediction is slower than other methods. Even though we added class 2-gram; the iPhone was still twice as fast as ours and some results become slower.

Table 8. Result of speed of input

\begin{tabular}{|l|r|c|c|c|r|}
\hline \multirow{2}{*}{\multicolumn{1}{c|}{ methods }} & \multicolumn{5}{|c|}{ Input speed (/min) } \\
\cline { 2 - 6 } & \multicolumn{5}{|c|}{ subjects } \\
\cline { 2 - 6 } & 1 & 2 & 3 & 4 & author \\
\hline Our word prediction & 41 & 29 & 42 & 41 & 81 \\
\hline Ours + class 2-gram & 35 & 33 & 34 & 57 & 85 \\
\hline iPhone & 84 & 65 & 79 & 67 & 77 \\
\hline Microsoft Office IME & 101 & 57 & 93 & 76 & 98 \\
\hline
\end{tabular}

\section{Examination}

Necessary numbers of input became lower than other methods by using our word prediction. We conjectured that using two different dictionaries and class 2-gram had a good effect on ours. According to the result, using next candidates and frequency change especially made our system $10 \%$ faster. From this, using a class 2-gram is effective to add to word prediction.

The result of speed of input was worse than other methods. The questionnaires we send out after the experiments showed that Linux line input makes our word prediction hard to see candidates. In actuality, only my result was better than others because I am familiar with it. It is conceivable that Kunrei-shiki Roman character input which was the trait of our word prediction made it difficult to input. Actually the questionnaires say that Kunrei-shiki is unfamiliar and it was difficult to confirm entered words.

\section{Conclusion}

We study new utterance support system working on an information terminal for the people who have a speech handicap. We use word prediction and added class 2-gram to it. The experience results show that our system needs less numbers of input but slower than other methods. Hereafter, we intend to create more effective dictionaries, improve class 2-gram and develop new GUI to make our system more effective and faster.

\section{Acknowledgment}

This work was partially supported by a Grant-in-Aid for Science Research (No.24500664) from the Japan Society for the Promotion of Science.

\section{References}

(1) Ministry of Health, Labour and Welfare : Survey on persons with physical disability, 4-25

(2) Glovalvoice : HOYA Service Corporation

(3) Toshiyuki Masui: "POBox: An Efficient Text Input Method for Handheld and Ubiquitous Computers. In Proceedings of the International Symposium on Handheld and Ubiquitous Computing”, HUC'99, 2-3, 1999

(4) SKK Openlab : http://openlab.ring.gr.jp/skk/dic-ja.html

(5) Morphological Analysis Software http://chasen-legacy.sourceforge.jp/

(6) Kanji Kana Simple Inverter: http://kakasi.namazu.org/

(7) Gregory W. Lesher :'Effects of Ngram Order and Training Text Size on Word Prediction”, 1-4, 1999 